\title{
La colección como dispositivo de lectura de la violencia política en la literatura infantil argentina
}

\author{
Laura Rafaela García *
}

Artículo recibido:

15 de abril de 2015.

Artículo aceptado:

9 de junio de 2015.

\section{Resumen}

Este artículo aborda el concepto de colección como un modo de leer y organizar un corpus de análisis a partir de temas u objetos determinados. El problema metodológico que implica seleccionar e interpretar los componentes del corpus de estudio se ve ejemplificado en este caso a partir del abordaje del tema de la violencia política de la última dictadura militar argentina (1976-1983) y la exploración de las representaciones de la violencia política en la literatura infanto-juvenil argentina. Proponemos realizar un recorrido por los puntos de contacto entre el campo de las memorias y los modos de apropiación del pasado a partir de los postulados de Elizabeth Jelin (2002), que define las

* INVELEC-UNT-CONICET, Tucumán, Argentina. lau2garcia@hotmail.com

INVESTIGACIÓN BIBLIOTECOLÓGICA, Vol.30, Núm.69, mayo/agosto, 2016, México, ISSN: 0187-358X. pp. 263-284 
narrativas del pasado como relatos comunicables que contribuyen a la construcción de sentidos para el futuro. Las colecciones de lecturas organizadas a partir de las formas de representar la violencia en los textos destinados a niños publicados entre 1970 y 1990 se presentan como un legado para las nuevas generaciones que no vivieron los hechos y, al mismo tiempo, muestra que la colección como dispositivo de lectura aun hoy interpela las prácticas de selección y organización de material de una zona literaria marginada por el sistema cultural.

Palabras claves: Colección; Tema; Violencia Política; Literatura infantil argentina.

\section{Abstract}

The collection as a reading device of political violence in children's literature in Argentina

Laura Rafaela-García

This paper discusses the concept of collection based on themes or objects as an approach to reading and organizing an analysis corpus. The methodological problem involving selection and interpretation of the objects of study is exemplified in the topic of political violence during Argentina's last military dictatorship (1976-1983) and representations of violence in children's literature in Argentina. On the bias of postulates forwarded by Elizabeth Jelin (2002), who defines stories of the past as communicable narratives that serve to build meaning in the future, we propose an examination of the contact points between the field of memory and modes from the appropriation of the past. Collections of children's readings published between 1970 and 1990 and organized in terms of the representation of violence stand as a legacy for future generations who did not experience the events. Even today the collection as a reading device challenges the selection and organization practices of material from this literary sub-genre existing at the margins of the dominant cultural system.

Keywords: Collection; Topic; Political Violence; Argentina Children's Literature. 


\section{INTRODUCCIÓN}

— $\mathrm{n}$ el prólogo del texto Oliverio junta preguntas de Silvia Schujer (2006) Ese presenta al protagonista, quien al principio se caracteriza por su actividad como coleccionista de preguntas. ${ }^{1}$ La búsqueda del joven es sencilla, pero la particularidad de su pasión hace que el personaje se distinga del resto porque, a diferencia de quien junta figuritas, Oliverio no puede comprar las preguntas en los kioscos, ni cambiarlas con nadie, y eso también muestra que ese álbum es infinito. Al principio juntaba sólo sus preguntas, después sus amigos empezaron a ayudarlo hasta que llegó un momento en el que las preguntas se repetían. El punto de estancamiento de la actividad de Oliverio, o en el que el coleccionista parece haber llegado al límite, se convierte dentro del relato en el desencadenante del cambio y el objeto coleccionado se resignifica cuando el personaje empieza a coleccionar respuestas.

Esta historia nos resulta interesante porque Oliverio es un gran coleccionista y el texto parece sugerirnos que quien colecciona se enfrenta a una tarea interminable. En esa dirección nos posicionamos para reflexionar sobre la tarea del lector como la de un coleccionista en una búsqueda inagotable de sentidos. En esta misma dirección que entiende al niño como un lector activo que entra en juego con el mundo de la literatura se inscribe el planteamiento de Walter Benjamin (1989), un apasionado coleccionista de libros. En "El panorama del libro infantil" el autor introduce dos ideas con respecto a la relación entre literatura e infancia que resultan claves para iniciar algunas reflexiones sobre la literatura para niños. ${ }^{2}$ Por un lado, se distingue la percepción de una experiencia en contacto con los libros en la cual el niño como sujeto lector es protagonista activo y, por otro, se alude a las múltiples manifestaciones de esa forma de itinerancia que aporta el auténtico pasaje por la literatura. Benjamin no sólo era un gran coleccionista sino que estaba particularmente atraído por esta literatura marginada, considerada para mujeres o para niños. Él se ocupaba de rescatar los viejos libros para la infancia como un legado del pasado y buscaba captar en ellos el deber de futuro.

1 "Oliverio coleccionaba preguntas como quien junta figuritas.

Preguntas de toda clase.

Grandes y chicas como: ¿Te gustaría saber por dónde queda el río por el cual el último barco fenicio pasó antes de que la civilización romana llegara a su fin? O bien: ¿Cómo te va? Fáciles y difíciles como: ¿De qué color era el caballo blanco de San Martín? O bien: ¿cuál es la raíz cuadrada de dos millones ochocientos cincuenta mil uno?...

Hasta que un día conoció a María Laura. O se le acabó la tinta. Y desde entonces, sin proponérselo, un nuevo cuaderno se le fue llenando de respuestas" (Schujer, 2006:10-11).

2 "En ese mundo permeable, adornado de colores, donde todo cambia de lugar a cada paso, el niño es recibido como actor. Con el ropaje de todos los colores que recoge al leer y mirar, se interna en una mascarada. Participa en ella al leer" (Benjamin, 1989: 73). 
Este trabajo se ubica en el cruce entre la memoria del pasado reciente y la literatura. El objetivo es reflexionar sobre los modos de leer en los espacios institucionales, en este punto el planteamiento trasciende el tema de la lectura en las escuelas y busca avanzar hacia los espacios de bibliotecas donde es posible delinear modos de leer que también dan cuenta de operaciones de selección y organización de los materiales de lectura. Para ejemplificar nuestra propuesta tomamos el tema de la violencia política y las formas de transmisión del pasado reciente a partir de lo ocurrido en la última dictadura militar argentina (1976-1983), para evitar que los hechos se repitan y sean olvidados por las próximas generaciones. Con este propósito rastreamos las referencias a la violencia política en la producción de la literatura argentina para niños y jóvenes entre 1970 y 1990. Proponemos con esto interpelar las prácticas literarias actuales que contemplan al niño como lector y, particularmente, pretendemos dejar planteada la cuestión sobre qué lugar ocupan los textos para niños en los estudios de la bibliotecología y en los archivos de acceso público.

Este trabajo forma parte de una investigación mayor que se propuso realizar un recorrido por los estudios de memoria, siguiendo los postulados de Elizabeth Jelin (2002) y por el campo infantil argentino. ${ }^{3}$ Esta exploración nos llevó a profundizar en las tensiones constitutivas de la literatura infantil y juvenil argentina y la marginalidad de esta zona literaria (Díaz Rönner, 2000), que en muchos contextos se ubica aun hoy en los bordes del sistema cultural.

En la selección de los textos del campo infantil argentino reconocemos una serie de relatos que interpelan al lector desde la ficción y que denominamos narrativas. Tomamos este concepto de los postulados de Jelin, quien logra capturar la importancia de estos relatos para la resignificación del pasado. Como señala la autora, la confluencia de determinados acontecimientos del orden privado enmarcados en contextos sociales compartidos impulsa la búsqueda de sentido. En este orden el acontecimiento rememorado o memorable será expresado en una forma narrativa, convirtiéndose en la manera en que el sujeto construye un sentido del pasado, una memoria que se expresa en un relato comunicable (Jelin, 2002: 27). Jelin atiende a dos elementos fundamentales: el pasado que cobra sentido en su enlace con el presente y la intención de comunicar esa experiencia que emerge de un proceso subjetivo.

Los textos de nuestro corpus aportan a los estudios de la memoria nuevas modulaciones para revisar el pasado desde las narrativas ficcionales. Estos

3 Nos referimos a la tesis doctoral Narrativas de la violencia politica en la literatura infantil argentina. Los trabajos de la memoria para contar la violencia politica (1970-1990) dirigida por Rossana Nofal (INVELEC-UNT-CONICET) y realizada en el marco de las becas de postgrado del Consejo Nacional de Investigaciones Científicas y Tecnológicas (CONICET). 
relatos tienen la particularidad de componer ciertas escenas o situaciones en las que los personajes y la acción misma abordan temas relativos a la vida política de la sociedad, como las relaciones de jerarquía, las formas de dominación y de resolución de los conflictos, la construcción de la legitimidad del poder, entre otros. El punto de partida de nuestra investigación fue la pregunta sobre cómo contar lo ocurrido durante la violencia política de la última dictadura militar argentina a las nuevas generaciones. Una forma de responder esta pregunta es organizar las colecciones de lectura e identificar en esta práctica la voluntad de transmitir y recordar el pasado a partir de las formas de representar la violencia en la literatura para niños.

En el campo infantil argentino se destaca un grupo de autores que emergieron a partir de la línea estética inaugurada en los años sesenta por María Elena Walsh, quien incorporó en su poética nuevas formas de apelar a la imaginación del niño desde el humor y la dimensión lúdica del lenguaje; vale mencionar también a Laura Devetach y a Elsa Bornemann. Entre los años sesenta y setenta este grupo de autoras y autores del campo infantil introduce en sus textos el elemento político por medio de la ficción, que contribuye a interpelar al lector sobre determinados aspectos de la vida social. No se trata de reconocer en los textos un conjunto de valores, ejercicio que implicaría hacer retroceder al campo hacia posicionamientos anteriores a los años sesenta, sino de mostrar por medio de las colecciones cómo los autores proporcionan herramientas al lector para conocer el mundo e interpelar al sujeto acerca de las formas de intervenir en él. El reconocimiento de estos desplazamientos en la narrativa del campo hacia la arbitrariedad de lo literario da cuenta del modo en que los autores articulan en la ficción lo empírico y lo imaginario, hecho que pone en evidencia una voluntad de transmitir cierta mirada sobre el mundo. En el campo infantil esa responsabilidad se manifiesta en la acción de contar, entendida como una práctica cultural de larga tradición en la literatura, por eso se abordan textos del género narrativo publicados en el periodo estudiado.

En este punto coinciden en nuestra investigación un tema particular relacionado con la transmisión del pasado y una zona pocas veces explorada por los estudios literarios, que dio lugar a reflexionar sobre la posibilidad de organizar colecciones de lecturas como una puerta de acceso al campo infantil argentino y un modo de dar a conocer las representaciones de la violencia política de un grupo de autores, que no sólo se distingue por su manejo del lenguaje simbólico sino también por aportar al imaginario colectivo. Al respecto, en pleno "boom de la memoria" en Argentina a principios del siglo XXI, Jelin destaca el mecanismo cultural por el cual la memoria tiene un papel fundamental para la construcción del sentido de pertenencia y lo 
relaciona con la actividad del coleccionista en un mundo que busca interpretar su pasado. ${ }^{4}$ El coleccionismo se presenta como una práctica cultural con vigencia en la actualidad y significativa para la construcción de la propia subjetividad.

En ese punto entender la colección como un modo de leer implica darle lugar a un interés particular del lector, que rastrea a partir de una inquietud propia las múltiples maneras de un tema o un objeto. Definimos la colección como un dispositivo de lectura por medio del cual cada sujeto organiza sus propios itinerarios y selecciona los textos que desde sus intereses personales aportan a la configuración de cierto tema. En esa operación de lectura, que tiene una base empírica, se distinguen al menos dos movimientos: uno de selección, por medio del cual el objeto se distingue del resto a partir de una característica particular, y otro de inclusión, en el que esa singularidad resignifica a un colectivo y aporta a un grupo mayor que a su vez lo distingue del todo.

La organización de una colección implica dos posicionamientos por parte del coleccionista: por un lado, la actitud de búsqueda que supone la acción de rastrear algo, que puede tener la forma de una pregunta que el sujeto lleva adelante y pretende responder con su actividad; por otro, la repetición de algo que llama la atención y que en su permanencia marca la continuidad o la unidad entre las partes que terminan por componer un todo. Del mismo modo, las colecciones tienen un lugar particular tanto en espacios públicos como en espacios privados, ya que fundamentalmente establecen vínculos entre distintas generaciones, se heredan y se conservan no sólo por el valor de sus componentes sino también por la carga afectiva, que les otorga un sentido especial.

Retomamos el personaje de Oliverio con su colección de preguntas, que es una figura representativa de lo que cada lector como heredero puede hacer con la lectura y la posibilidad de armar sus propias colecciones de textos. En ese encuentro entre el texto y el lector se desencadenan múltiples sentidos y alternativas, como en el caso del personaje de Schujer, que transforma su colección inicial de preguntas insólitas en una colección de respuestas como una forma de dar lugar a su posibilidad de reapropiarse de los objetos. En es-

4 "Vivimos en una era de coleccionistas. Registramos y guardamos todo: las fotos de infancia y los recuerdos de la abuela en el plano privado-familiar, las colecciones de diarios y revistas (o recortes) referidos a temas o períodos que nos interesan, los archivos oficiales y privados de todo tipo. Hay un culto al pasado, que se expresa en el consumo y mercantilización de diversas modas "retro", en el boom de los anticuarios y de la novela histórica. En el espacio público, los archivos crecen, las fechas de conmemoraciones se multiplican, las demandas de placas recordatorias y monumentos son permanentes. Y los medios de comunicación estructuran y organizan esa presencia del pasado en todos los ámbitos de la vida contemporánea." (Jelin, 2002:10) 
te sentido, tomamos el concepto de apropiación de Analía Gerbaudo (2011), que parte de los conceptos de Jacques Derrida para el discurso literario, y reflexionamos sobre las posibilidades de entender al lector como un heredero capaz de resignificar los textos y sus búsquedas de significado. ${ }^{5}$ Las narrativas de la literatura infantil y juvenil en estas colecciones se presentan como un legado que inicia un camino no previsible en la experiencia subjetiva del lector en relación con su tiempo y su espacio y aportan a la construcción de nuevos sentidos del pasado.

\section{LA COLECCIÓN COMO DISPOSITIVO DE LECTURA}

Para organizar una colección es importante partir de la voluntad de reunir un conjunto de elementos con base en un eje vertebrador, que puede ser un tema o un objeto a determinar en el recorrido por cierto campo, tema o recorte temporal en torno al cual todo un colectivo de objetos cobrará sentido. Conviene aclarar que el lector experto tendrá un ritmo distinto de apropiación y organización de los relatos en colecciones al de un lector que recién se inicia en un campo y en la práctica, debido al tiempo que pueden llevarle las actividades de recopilación y selección.

El concepto de tema es operativo para organizar la colección en el caso de los textos literarios. En este punto seguimos a Raymond Williams en Marxismo y literatura, cuando plantea este término como una variable determinante, que junto con la posición o la elección de un modo de organización del texto y el modo de composición formal definen el concepto de género (1980: 210). Como rasgo constitutivo de la categoría de género, el tema es una variable relacionada con las distintas formas del proceso material social y del sistema literario; por lo tanto, surge del campo de acción o de la cualidad del objeto o de un proceso en particular.

Williams destaca que el tema está sujeto a las variaciones sociales, culturales e históricas, de modo que la emergencia de los estudios sobre la memoria del pasado reciente parte de la revisión de los horrores de la dictadura y su enfoque interdisciplinario que hace posible el cruce con la literatura. En nuestro caso, esto motiva la pregunta por la forma en la que los autores aluden a la violencia política en pleno contexto dictatorial y qué tipo de referencias es posible reconocer incluso después, durante los primeros años de la democracia.

5 Gerbaudo lo expresa en los siguientes términos: "Hereda, o es digno de considerarse un heredero quien en parte es infiel, es decir quien se apropie, quien pueda hacer algo nuevo con aquello que trae" (2011: 22). 
En este sentido, nos interesa resaltar que la colección como modo de leer está determinada por las características del problema que nos ocupa y la definición del tema es el punto de partida para organizar el corpus de trabajo de la investigación. La colección se ajusta a la naturaleza fragmentaria del campo de las memorias para dar cuenta de las heridas del pasado, sus interferencias y fracturas como también de las formas de crear nuevos sentidos para el futuro. Al mismo tiempo, esa misma naturaleza fragmentaria hace soportable el relato del pasado. En la marca subjetiva de la colección se inscribe el proceso de la transmisión. Jelin y Kaufman (2006: 9) afirman que la subjetividad refiere a procesos y dinámicas que constituyen lo propio de la existencia humana: dar sentidos y crear sentidos, articular de manera singular y única experiencias, representaciones y afectos. Las especialistas en memoria sostienen que ese proceso es individual y también social, porque las experiencias y afectos están siempre inmersos en lazos sociales.

Por otra parte, la profusión de temas y textos en la literatura argentina para niños de las últimas décadas es tal que es posible abordar el campo a través de la colección como dispositivo alrededor del cual organizar distintas colecciones sobre diferentes temáticas. La colección aporta al modo derrideano de entender la lectura que según Gerbaudo se presenta como una travesía que se abre con el texto que se elige, pero que conduce hacia otros textos, hacia las representaciones que guarda la memoria de otros textos, sin que sea posible determinar con certeza la totalidad de los textos involucrados en el trabajo (Gerbaudo, 2007: 259). Desde esta concepción es posible ordenar distintos itinerarios a partir de los objetos o del hilo de la trama que se decida seguir. La organización de colecciones responde a otro de los intereses de nuestra investigación, que es dar a conocer los modos de la ficción para abordar la violencia política en un campo tan ignorado como prolífico como es el de la literatura infantil y juvenil. En esta dirección, para darle continuidad a la lógica de la colección como una posibilidad de explorar y conocer el campo, podríamos indagar sobre las representaciones de la infancia en los textos para niños, las manifestaciones del miedo o las modulaciones de la figura del animal para proponer algunas temáticas factibles y atractivas a la lectura y el conocimiento de esta zona literaria.

Con respecto a la identificación y construcción del corpus en las investigaciones literarias es fundamental el aporte de Miguel Dalmaroni (2009), quien al determinar el corpus como un dispositivo de dominio explicita algunas preguntas que aportan en la dirección de nuestros planteamientos. ${ }^{6}$

6 “El orden del conjunto es la condición para reconocer una “colección”? ¿Quién colecciona, y en base a qué criterios?” (Dalmaroni, 2009: 70) 
Esta operación implica inevitablemente un ejercicio de violencia simbólica, ya que supone mecanismos de dominación y exclusión que se inscriben en la histórica disputa de canon y corpus e introduce el diálogo con autores de otros tiempos y se pregunta qué colección ordenaron determinados sujetos de un cierto pasado (Dalmaroni, 2009: 74). Por último, la disyuntiva filosófico-epistemológica nos enfrenta al problema que implica construir un corpus histórico-narrativo y crítico, que busca producir y experimentar algo nuevo. En nuestro caso, para explorar las representaciones de la violencia política en el campo infantil argentino nos posicionamos como críticos con el propósito de aportar nuevos sentidos para resignificar el pasado, sentidos que trasciendan el discurso histórico o testimonial de los hechos. Pero, a su vez, nos posicionamos como lectores en busca de ciertas huellas o referencias soterradas que nos permitan dar respuesta a la pregunta inicial de nuestra investigación, que es cómo contar los horrores de la dictadura a las generaciones que no vivieron los hechos.

Para organizar el corpus de nuestro trabajo también partimos de la lectura del artículo "Literatura para chicos y memorias: colección de lecturas" de Rossana Nofal (2006), quien organiza la primera colección para contar la violencia política a partir de una serie de relatos para niños reunidos en torno al concepto de fantasy literario de Rosmary Jackson (1986). Este trabajo se presenta como un recorrido personal, en el que la autora expresa su experiencia como lectora del campo infantil y su inquietud sobre las formas de inscripción de la última dictadura en la literatura infantil y juvenil argentina. ${ }^{7}$ Entre los puntos que consideramos para nuestro análisis tenemos el objeto como denominador común de una colección -en nuestro caso se trata de relatos-determinado por la subjetividad del coleccionista, la singularidad de lo individual y el aporte al conjunto que integra; así como la marca particular que el coleccionista le imprime al objeto al seleccionarlo para su colección donde coinciden, una vez más, el valor subjetivo y el posible movimiento de ese objeto de un lugar marginal para ocupar un nuevo lugar entre lo extraño o lo exclusivo.

Estas condiciones motivan nuestra propuesta para revisar las narrativas de la literatura infantil, ya que los autores del campo interpelan desde la literatura al niño y desafían lo esperado por los adultos en cuanto a las temáticas y los géneros. El primer movimiento consiste en rastrear en este campo las figuras que hacen trasmisible el pasado reciente y potenciar en la reunión de

7 Con respecto a la organización de las colecciones Nofal aporta algunas claves: “¿Qué hace que un conjunto de objetos se convierta en una colección? La coherencia e individualidad de cada elemento, la misma clase, las técnicas y lógicas para ordenarlos, la norma, el interés por el valor especial y la seducción de lo extraño”. (2006: 115) 
las narrativas las posibilidades del campo infantil para tratar el tema de la violencia política. Entendemos que cada uno de estos relatos encuentra su lugar en la colección y en ese movimiento de localización se distinguen por su forma particular de aludir a la violencia. Pero, a su vez, podrían formar parte de otras series porque los temas abordados y las historias presentan esa posibilidad. En cada relato reconocemos la marca singular de un autor y como lectores encontramos en la ficción una modulación del pasado. En ese gesto el relato adquiere dimensión pública al formar parte de la lógica colectiva de la colección. Es allí donde se produce el movimiento de interpretación que aporta a la construcción de sentidos para reflexionar acerca del tema en cuestión.

La confluencia de los planteamientos de Jelin para resaltar la necesidad de apropiación del pasado reciente y resignificar los relatos, la organización de un corpus que represente un nuevo aporte para la investigación literaria -como plantea Dalmaroni-, la lectura como parte de una experiencia que surge del recorrido personal por un campo, lo inabarcable de la saturación semántica y la posibilidad de interpretar el trabajo del lector o del crítico como un heredero infiel de largas tradiciones -como sugieren Nofal y Gerbaudo- contribuyeron a la organización de tres colecciones de lecturas desde las que consideramos posible contar la violencia política en el discurso literario.

\section{ITINERARIOS DE ELEFANTES, SAPOS Y MONSTRUOS}

Hemos afirmado que el concepto de tema es fundamental para organizar un recorrido por las lecturas del campo que dan lugar a la conformación de una colección. Pero también son importantes los objetos o figuras porque por medio de la repetición de ellos en los textos dentro de cierto recorte temporal es posible ubicarlos en una serie y distinguir la importancia de su presencia y de su continuidad. En este apartado nos proponemos presentar un breve panorama de las colecciones, pondremos el acento en algunos momentos claves de su organización y revisaremos en términos generales los textos que se incluyen en cada una de ellas con el objetivo de ejemplificar algunos posibles movimientos de la composición. ${ }^{8}$

La primera colección se denominó "memoria de elefante", estableciendo un juego con el dicho popular que destaca la importancia de recordar y la buena memoria que distingue a los elefantes. Esta figura aparece como mo-

8 Estas colecciones fueron desarrolladas en detalle tanto en el planteamiento de la tesis doctoral como en distintos artículos publicados (García, 2010; 2012; 2013) y discutidos en diferentes espacios especializados. 
tivo repetido en una serie de relatos del periodo estudiado. El primer texto que llamó la atención fue el cuento "Un elefante ocupa mucho espacio" de Elsa Bornemann (2008 [1975]), ${ }^{9}$ que forma parte de uno de los libros para chicos que fueron prohibidos durante la última dictadura, ya que representaba una amenaza para los valores morales y cristianos que el régimen dictatorial decía defender. La lectura de este texto y la importancia del personaje central, que promueve la rebelión de los animales del circo a los maltratos de los domadores, fueron el disparador para reunir en torno a esta figura una serie de relatos siguiendo el orden de aparición.

Por el simbolismo de sus historias y el protagonismo que cobra el elefante en los textos esta colección se basa en la metáfora como recurso retórico que alude a las formas políticas del mundo adulto. El cuento mencionado nos llevó a revisar en los textos anteriores del campo y encontramos la primera historia de un elefante en la novela de María Elena Walsh titulada Dailan Kifki (2007 [1966]). En este caso el elefante es la mascota que la protagonista quiere adoptar y a partir de allí se desata esta disparatada aventura, que desafía el orden institucional de la sociedad y expone en tono de parodia las dificultades del adulto para responder a esta situación.

Luego, encontramos el cuento "Guy" de Laura Devetach, incluido en Monigote en la arena (2008 [1975]), que toma como motivo el miedo a desaparecer del elefante cuando ve diluirse su reflejo en el agua. Este cuento establece una provocadora relación con el contexto de producción, en el que empezaban a darse las primeras desapariciones físicas de personas como mecanismo de exterminio aplicado durante la dictadura. En tercer lugar-siguiendo un orden cronológico para ordenar la colección- se ubica Víctor, el protagonista del cuento "Un elefante ocupa mucho espacio", que está ligado al texto anterior porque el título recupera la frase que repite Guy para expresar su miedo a desaparecer. La propuesta de Víctor tiene que ver con la vida en libertad de la selva, que la mayoría de los animales del circo no conoce. En clave de ficción, hay una propuesta social para revertir la situación de opresión que atravesaba la sociedad argentina en general en ese momento.

En 1984, durante la democracia, Gustavo Roldán publica el cuento “¿Quién conoce el elefante?” en El monte era una fiesta (2008a). En la conversación entre el Sapo y la Vizcacha este texto recupera la figura del elefante y algunas de sus características, que en la lógica de la colección cobra sentido y puede entenderse como la voluntad de reponer esta figura a partir de la censura del texto de Bornemann. A éste le sigue otro cuento del mismo au-

9 Aclaramos que en adelante incluimos entre corchetes la fecha de la primera publicación de los textos en función del recorrido histórico que proponemos en este trabajo y la importancia del contexto de producción. 
tor, titulado "Prohibido el elefante" (Roldán, 1999 [1988]), en el que se da el enfrentamiento entre el grupo del Puma, que decía que el elefante era del tamaño de un caballo, y el grupo del Jaguar, que sostenía que era del tamaño de un ratón. Este hecho se resuelve por medio de las elecciones, en las que debido al empate se concluye de manera unánime que el elefante no existe. A lo largo del texto vemos la reacción de la Pulga, que verdaderamente conoce al elefante porque vivió en el circo, pero a quien le prohíben hablar por su tamaño y por la versión que sostiene en contra de los animales más poderosos del monte.

Por último, incluimos en nuestra primer colección al cuento "El genio del basural", que pertenece a El héroe y otros cuentos de Ricardo Mariño (2008 [1995]); en él se narra la historia de un niño que vive en un basural y encuentra una tetera oxidada, como una especie de lámpara mágica, de la que sale un genio malhumorado, a quien el chico le pide como primer deseo un elefante. Esa presencia genera una serie de aventuras en un mundo plagado de carencias materiales en contraste con la exótica presencia del elefante. En el cierre de este recorrido este cuento simboliza la voluntad de memoria como una elección del sujeto para apropiarse del pasado. Si se avanza con mayor detalle en las situaciones narrativas de los textos incluidos en esta colección es probable descubrir la carga simbólica de cada uno de los elefantes, que suman a la alegoría de las posibilidades e imposibilidades de los hombres para actuar ante el autoritarismo, las diferencias ideológicas y las jerarquías, etc. como así también la importancia de las acciones colectivas, sin apelar a la subordinación y al sometimiento del otro. Se trata de prácticas políticas que atraviesan la vida social y la figura del elefante potencia y multiplica a medida que se avanza en el recorrido.

La segunda colección tiene como elemento unificador al Sapo, un personaje de los cuentos de Gustavo Roldán que se caracteriza por tener un vínculo privilegiado con el pasado. Él es el narrador del grupo y su principal herramienta es la palabra; por lo tanto, dentro del colectivo de animales que componen el monte chaqueño el Sapo se posiciona como la voz autorizada y representativa del conjunto de animales. En el prólogo de Cada cual se divierte como puede (Roldán, 2007a [1984]) el Sapo interviene para denunciar al autor y reclamar la autoría de las historias que viene contando Roldán. Reconocemos en ese gesto la identificación o proyección del autor en el rol de narrador que desempeña el personaje del Sapo a lo largo de su poética y le permite posicionarse como nexo entre pasado y presente para abordar el tema de la violencia y otros más a partir de su experiencia. En esta colección distinguimos una primera parte de relatos que apuntan a describir y jerarquizar el lugar del Sapo como mediador entre la vida del monte y la ciudad, entre el pasado y el 
presente; y luego, una segunda parte en la que se alude a las formas de la violencia política.

El primer cuento en el que el Sapo hace su aparición es "Sobre lluvias y sapos”, allí se presenta como la víctima de la superstición de otros animales, quienes para lograr que llueva lo tienen prisionero panza arriba. Este relato se basa en el diálogo entre varios personajes, recurso que resalta el predominio del estilo oral y se mantendrá como rasgo distintivo de la poética del autor. El tono particular de esta interacción está dado por el contrapunto de voces, especialmente, sostenido por el Sapo que logra escapar gracias a su astucia. El segundo texto que contribuye a la construcción del personaje también aparece como el anterior en El monte era una fiesta (Roldán, 2008a [1984]), el cuento que lleva el título del libro traza la geografía del monte dividido en dos por el río, en cada uno de sus lados conviven animales chicos y grandes. El narrador habla desde "éste lado del río" y a medida que se avanza en la lectura de los cuentos se conoce cómo es la vida de este lado de la orilla. En “¿Quién conoce un elefante?” se manifiesta su capacidad de representar y se muestra también la influencia de su palabra para colaborar en la construcción del imaginario colectivo del monte, pues a medida que describe al elefante los otros animales reconstruyen su apariencia. Por otro lado, el tercer cuento importante de este texto es "Lluvias eran las de antes", aquí el Sapo nos remonta a su origen y el recurso para legitimar su autoridad y su saber es su experiencia de vida, que data de su participación en el diluvio universal.

Por otro lado, el cuento "Un monte para vivir", incluido en Cada cual se divierte como puede (Roldán, 2007a [1984]), deja ver el proyecto colectivo en el que se inscriben los personajes dentro de esta poética. Encontramos las primeras referencias a la violencia política, como la prohibición y la persecución que genera la ley impuesta por el tigre, a la que muchos animales se adhieren. Esto da como resultado el silencio y el miedo en el monte entre algunos de sus habitantes, que prefieren irse a vivir al otro lado, como una forma de representar el exilio. Otro cuento importante para esta colección que revela la importancia de la palabra del Sapo como mediador del diálogo intergeneracional es "Como si el ruido pudiera molestar" (Roldán, 2007b [1986]). Como buen narrador el Sapo es quien habla sobre la muerte del Tatú a los animales más chicos del monte, el cuento que lleva el título del libro alude a la incomodidad de la muerte y el vacío que provoca el silencio de la ausencia. El Sapo muestra en su relato la hostilidad de la muerte como una parte de la vida, la despedida del personaje está presentada desde la rememoración, la alegría de los mejores recuerdos y las aventuras compartidas por todos con el Tatú. 
"El tamaño del miedo" relata el encuentro del Coatí con el miedo. En la primera parte el narrador usa el suspenso como estrategia del contrapunto oral porque el Coatí nunca dice quién lo perseguía, pero describe a su contrincante mientras responde a las preguntas del Sapo. Éste descubre que el monstruo no era ningún animal, que era producto de la imaginación del Coatí y entonces, con astucia, lo ayuda a desarmar la imagen de ese monstruo y a enfrentarlo. Todo el auditorio repleto de animales pregunta cómo lo reconoció y el Sapo cuenta que se enfrentó muchas veces con él. La historia cierra con el Sapo asegurando sabiamente que el miedo de cada uno es como el miedo de cada uno. En esa escena la subjetividad se presenta como parte de la construcción de cada sujeto para enfrentar los desafíos y es aceptada como un rasgo de la identidad personal y compartida de manera colectiva.

Uno de los textos fundamentales de Roldán para abordar la violencia política es Sapo en Buenos Aires (Roldán, 2008b [1989]), que incluye dos cuentos importantes: "Gustos son gustos" y "Las reglas del juego". En el primer relato el Sapo describe la vida en la gran ciudad y les cuenta a los animales del monte el apuro cotidiano, el amontonamiento, la basura en el río, el interés por conocer los animales de otros países, etc. como elementos que representan el color local urbano. La alusión a las prohibiciones y a la intervención policial como parte de un modo de vida muestra también una crítica al funcionamiento social y a un orden ilógico, aludiendo indirectamente a las múltiples formas de censura. El Sapo naturaliza la arbitrariedad de las medidas que afectan a los habitantes de la ciudad y los animales del monte no encuentran explicación y se indignan ante la impotencia de la respuesta del Sapo, que da título al cuento.

En "Las reglas del juego" se habla de la organización política de la ciudad. El juego, llamado Elecciones, tiene varios momentos: hacer pegatinas por las paredes de la ciudad, votar y algunas otras actividades que supone el tiempo electoral. El Sapo mantiene la objetividad para explicar el juego a pesar de la incomprensión de los animales que reaccionan contestatariamente ante el abuso permanente que se expresa en el relato. Cuando describe a los militares el Sapo recurre a un código compartido y logra transmitir la dimensión del peligro de su presencia a los animales del monte. El Sapo les cuenta que había muchas personas que no estaban de acuerdo y andaban tratando de cambiar el final y las reglas del juego como una alternativa a la situación.

Como conclusión de los relatos recorridos, entendemos que los planteamientos de los animales del monte ponen de manifiesto el funcionamiento de un sistema de normas o códigos diferentes en torno a un proyecto ético y político de la vida del colectivo de animales. Es evidente que en la poética de Roldán la violencia política encuentra una alternativa para ser superada 
por medio de las acciones colectivas. Esta colección para contar la violencia política se construye a partir de un recurso como la analogía, que permite trazar similitudes entre dos organizaciones sociales representadas por la vida misma en el monte y por lo que pasa del otro lado del río. En ese contexto el mediador entre las distintas partes y los diferentes tiempos es el Sapo. Roldán muestra situaciones similares a las de la realidad social argentina y las traslada en sus relatos a la vida del monte para resaltar irónicamente las posturas asumidas así como las variantes para resolver los problemas.

La tercera colección se organiza en torno a la figura del monstruo y sus posibles modulaciones, por lo que después de analizar la composición de la colección decidimos darle el nombre de "lo monstruoso". Los textos incluidos se publican entre fines de la década de los ochenta y la primera mitad de los noventa, es decir, corresponden a los primeros años de reorganización democrática. Nos detenemos en el contexto por dos razones: por un lado, permite resaltar los movimientos del campo infantil que con estos textos incluye las primeras aproximaciones del género fantástico en la literatura infantil argentina; por otro, uno de los temas centrales en la opinión pública en ese momento eran los reclamos de justicia por la desaparición de personas durante la última dictadura. En este punto, parte del clima de la época muestra que la sociedad no sólo se vio afectada por la experiencia misma de la violencia sino que también las voces de las víctimas y afectados directos movilizaron la sensibilidad de ciertos grupos sociales. Recordemos que por estos años tiene lugar la conformación de la Comisión Nacional de Desaparición de Personas (CONADEP), que como parte de ese trabajo publica en 1984 el informe titulado Nunca Más, que reúne los testimonios de sobrevivientes y testigos de los centros de exterminio.

Con lo monstruoso -que resulta de la apropiación de lo fantástico y, en particular del fantasy en la literatura para niños- aludimos a una zona narrativa que abarca temas y situaciones relativas al extrañamiento que produce la deformación de lo familiar en el sentido de lo siniestro de Freud, la participación de monstruos y fantasmas como protagonistas que recrean "lo otro", distante y cercano a la vez del mundo del lector, y la sensibilidad que trasciende el relato para dar lugar a una experiencia subjetiva construida en los límites de lo indecible y lo representable. La singularidad de la experiencia de la violencia influye en los autores del campo y éstos recurren al fantasy literario como género que subvierte y ataca el orden cultural de lo establecido al deshacer sus estructuras y significaciones.

Para definir el concepto seguimos a Rosmary Jackson (1986), quien en su trabajo toma tres características centrales del fantasy: la forma en la que perturba y pone en cuestión lo representable y "lo real" en la literatura; la 
hostilidad del fantasy hacia las unidades estáticas, la yuxtaposición de elementos incompatibles y su resistencia a la fijación; por último, la disolución de las nociones básicas de tiempo, espacio y personajes, la apropiación del lenguaje y de la sintaxis que apuntan al cuestionamiento del orden social y el sentido de la vida (Jackson, 1986: 12-13). Las modulaciones del fantasy hacen posible abordar temas relativos a la fragmentación de la personalidad, el miedo natural a lo informe, la ausencia, la muerte, y también presenta una forma de resistir o reaccionar contra el orden social arbitrario.

Lo monstruoso reconocible en la singular figura del monstruo asume la forma simbólica de los miedos en los relatos que hablan desde la posición de éste o de lo que éste provoca. Este último aspecto del tema está representado en el primer cuento de nuestra colección, titulado Irulana y el ogronte (Un cuento de mucho miedo) de Graciela Montes (1991), que se construye sobre la oposición entre el tamaño superlativo del monstruo y la pequeñez de la protagonista del relato. El recurso oscila entre dos manifestaciones del miedo, pues mientras que la protagonista se paraliza al ver al monstruo y los desmanes que provoca su malhumor, el pueblo debe subordinarse también al estado de ánimo del monstruo. La arbitrariedad del poder representada en este cuento es una de las modulaciones posibles para abordar no sólo la violencia política sino también temas como la relación entre adultos y niños. Al principio de la historia nos enteramos que el nombre de la protagonista es Irenita, pero gracias a su valentía y la forma en la que su nombre interviene en la historia la narradora le pone el apodo de Irulana. En la escena del final Irulana se encuentra frente al monstruo dormido, en plena oscuridad de la noche, y corriendo los peores riesgos se anima a gritar bien fuerte su nombre. La importancia de esta escena y del poder de la palabra, que justamente en este caso es el nombre propio, aporta más sentido a las escenas reunidas en esta colección, que también deja ver algunas claves para enfrentar los miedos.

La contracara del monstruo aparece parodiada en esta serie en el prólogo de ;Socorro! (12 cuentos para caerse de miedo) (Bornemann, 2004 [1988]), que está firmado por el mismo Frankenstein. En la poética de Elsa Bornemann los modos de abordar el terror están mediados por el humor y, en este caso, la parodia es introducida por la referencia intertextual con este personaje clásico del género gótico. Con tono amigable Frankenstein aprovecha el espacio cedido por la autora para confesar sus miedos y manifestar su deseo de ser querido. La humanización del personaje es el recurso para apelar al lector y a su elección por las lecturas de terror. El texto ;Socorro! (12 cuentos para caerse de miedo) da cuenta de lo tormentoso que pueden ser los miedos. El miedo a la muerte que nos persigue desde niños y, en este caso, es abordado en diferentes situaciones relacionadas con la niñez: una abuela perversa 
que después de muerta cobra vida en los objetos heredados y termina por hacer desaparecer a su única nieta mujer en el cuento "La del Once Jota" o la metamorfosis de "El Manga", un extraño vecino que se transforma en una araña y secuestra a los niños para atraparlos y matarlos en su tela. La animación de objetos inanimados, el desdoblamiento, la transformación y la multiplicación de los personajes son algunos de los motivos del fantasy literario que aparecen a lo largo de los cuentos de este libro y parten de elementos reales o situaciones cotidianas para generar cierta ambigüedad e interpretar el mundo desde las propias impresiones del sujeto.

Otra inflexión de esta colección es una de las primeras novelas cortas del campo infantil titulada Tengo un monstruo en el bolsillo de Graciela Montes (2003 [1988]). Inés, la protagonista, proyecta sentimientos y deseos en el monstruo que tiene en el bolsillo de su delantal. La niña narra en primera persona los hechos y presenta situaciones que con el transcurso de la historia dejan ver cómo se enfrentan y resuelven los miedos personales relacionados con la apariencia física, la timidez y las dificultades que le ofrece su entorno escolar y familiar en el momento de transición a la adolescencia. En este caso, el monstruo funciona como una proyección de los pensamientos de Inés y reacciona de distintas maneras. Al principio, Inés estaba un poco sorprendida y al mismo tiempo se divertía con el monstruo que crecía y se achicaba a su antojo. Sin embargo, llegó un día en el que quiso liberarse del monstruo, que cada vez ocupaba más y más espacio con todo lo que destruía y como no pudo sacárselo de encima tuvo mucho miedo. La historia se resuelve cuando ella logra enfrentar determinadas situaciones personales y se anima a contarle a su abuela Julia el secreto del monstruo. Ésta lo toma como algo natural, le dice que todos tienen un monstruo escondido y que el mejor método para achicarlo es hablar de él.

Otra faceta de la figura del monstruo aparece en la novela Maruja de Ema Wolf (2011 [1989]), que parodia la vida de los monstruos. El texto cuenta la historia de Veremundo, un horroroso y destartalado monstruo que odia a los niños. Todo empieza un día que irrumpe en su hogar su tía fantasma, llamada Maruja. Ésta se instala súbitamente en la mansión de su sobrino debido a una inundación en su barrio del cementerio. La experiencia de este monstruo es la de la invasión del espacio personal y de su forma de vida, la visita de su tía le trae una serie de consecuencias y lo peor es que, a pesar de sus intentos, todo hace pensar que es casi imposible que Maruja vuelva al cementerio. La parodia de situaciones muy cercanas a las reales en la vida de los personajes interpela al lector que pasa de solidarizarse con el monstruo a divertirse con las ocurrencias de su tía, que es una caja de sorpresas. 
En esta colección también encontramos textos que de manera menos ficcional muestran el poder perturbador de lo secreto y lo siniestro en las modulaciones subjetivas de la experiencia de la violencia, al mismo tiempo que instalan temas silenciados en la sociedad en ese momento. El cuento de Bornemann titulado "Nunca visites Maladony", incluido en ;Socorro! (12 cuentos para caerse de miedo) (2004 [1988]), narra la historia de Timothy Orwell, un joven de trece años a quien misteriosamente un día desconocen sus vecinos y su propia familia. Al tiempo que el lector busca una explicación para comprender el extrañamiento hacia el joven el relato avanza en la ambigüedad de un mundo familiar que abruptamente se vuelve ajeno y hostil.

Cuando Tim llega a su casa después de la escuela no encuentra ninguna referencia a su mundo familiar. Entonces, los nuevos dueños de la casa y los vecinos lo creen loco. El joven se despierta en la sala de un hospicio, atado a la cama, y como en una pesadilla las personas que lo cuidan son los miembros de su familia. Allí pasa cuarenta años hasta que le dan el alta cuando deja de identificar como familia a las personas que lo rodean, porque ya estaban todos muertos. La narradora, una estudiante argentina que iba por una beca a Londres, hacia el final interpela a Tim, quien le cuenta la historia y le da su propia explicación de los hechos. Después Tim desaparece entre la multitud londinense y la narradora decide volver a su país. De modo que hacia el final se deja planteada la paradoja de la situación en una alusión directa a la fecha en la que ocurren los hechos (1978), en el momento más violento de la dictadura. La sensación de extrañamiento en la que se construye todo el relato es también una modulación de la invasión violenta que altera la vida cotidiana del protagonista, que sufre el encierro y el olvido o el rechazo de su comunidad.

El último texto es la novela Otroso. Últimas noticias del mundo subterráneo de Graciela Montes (2007 [1991]), donde se manifiesta la necesidad de transgredir el orden de lo real por medio de la representación del mundo subterráneo. En este relato se reafirma el orden institucional de la vida clandestina de un grupo de jóvenes y el de la violenta amenaza que representa la Patota. Este orden establece posibles referencias con la militancia en los años setenta y el procedimiento de las fuerzas armadas durante la dictadura. Además, estos elementos se mezclan con el discurso metaliterario introducido por el narrador para contarnos cómo arma la historia y su relación con los testigos que después de un tiempo se animan a hablar. La importancia de este texto radica en la forma en la que representa las débiles y borrosas fronteras entre lo privado y lo público que se ven amenazadas por la violencia, abordando temas como la clandestinidad y la persecución, para lograr un clima de permanente intimidación. 
Este recorrido general por los temas de lo fantástico en la literatura para niños deja ver que la violencia puede contarse también desde las manifestaciones subjetivas en las que se experimenta el miedo, el silencio, el olvido y el extrañamiento en determinadas escenas. El recurso sobre el que se organiza esta colección es el de causa-consecuencia, la primera es el miedo y da como resultado las múltiples manifestaciones de la subjetividad trabajadas desde las modulaciones del fantasy.

Como conclusión del recorrido por nuestras colecciones podemos afirmar que la literatura no trata de aludir directamente a los hechos violentos del pasado -de eso se ocupan otro tipo de discursos- sino de interpelar la sensibilidad del lector por medio de situaciones que dan cuenta de las formas en las que la violencia incide en el sujeto y, por lo tanto, el planteamiento de los textos trasciende su contexto de producción y el tema de la violencia política. Como afirma Nofal (2006), en la literatura infantil la violencia es comunicable por medio de la fantasía. En el caso de nuestras colecciones podemos reconocer tres claves de lectura para narrar el pasado reciente: las arbitrariedades del poder y el develamiento de las formas políticas del mundo adulto que se pone en evidencia en ciertas escenas de la primera y la segunda colección; la potencialidad de las acciones colectivas lideradas por el Sapo y el revolucionario pensamiento del elefante que dan lugar al cambio y al diálogo intergeneracional y, por último, la experiencia del miedo como una modulación de las heridas privadas y los traumas públicos de la violencia política.

El pacto de ficción asumido al iniciar la lectura de un texto literario y la continuidad de las figuras propuestas por cada colección contribuye a la construcción de la subjetividad del lector, de modo que durante la lectura intervienen planteamientos que permiten al lector identificarse con uno u otro personaje, situación o tema. Además, como lectores, sabemos que después de la lectura la mirada del sujeto no es la misma. En esta dirección, el recorrido por las poéticas de autor y los textos reunidos en nuestras colecciones ponen al lector frente a nuevas condiciones para interpelar al mundo y definir su forma de participar en él.

\section{CONSIDERACIONES FINALES}

A partir de lo planteado, concluimos que en la literatura se ensayan acciones, valoraciones y consideraciones que entran en disputa en la subjetividad del lector y en el acto de leer, generando un espacio de múltiples variaciones imaginativas (Ricoeur, 1999). La relación que planteamos entre la lectura de colecciones, la continuidad de un tema u objeto y los procesos subjetivos en 
juego para crear sentidos encuentra en la colección un dispositivo de lectura que cobra un sentido particular en relación con el tema planteado pero al mismo tiempo permite el abordaje de diversos temas.

La literatura puede provocar un acontecimiento al abrir la interpretación y dar a leer, por eso es posible abordar algunas zonas ficcionales de la literatura para niños en las que los autores conceden al lector una forma de interrogar al mundo por medio de la lectura. Los cuentos y las situaciones narrativas que componen estas colecciones articulan una experiencia y una voluntad de legar por medio de la lectura. En este punto la práctica del coleccionismo tiene filiación con la transmisión entre las generaciones y con un contexto actual en el cual el individuo tiene una necesidad de inscribir su propia marca. Según Jelin,

para poder transmitir los sentidos del pasado hay al menos dos requisitos: el primero, que existan las bases para un proceso de identificación, para una ampliación inter-generacional del "nosotros". El segundo, dejar abierta la posibilidad de que quienes "reciben" le den su propio sentido, reinterpreten, resignifiquen -y que no repitan o memoricen-. (2002: 126)

En la misma dirección, Jacques Hassoun asegura en Los contrabandistas de la memoria (1996) que todos somos depositarios y transmisores de aquello que nos han legado, ya que el paso de una a otra generación supone la construcción de una transmisión en tanto actividad y no es algo que ocurre naturalmente. Los autores del campo infantil a través de estos relatos funcionan como mediadores entre pasado y presente, tienen un rol como agentes sociales y, en muchos casos, aprovechan su vínculo privilegiado con el pasado y su concepción del niño como sujeto capaz de ganar autonomía en contacto con la literatura para apelar a ellos desde la imaginación.

La lectura de la colección como dispositivo de abordaje metodológico revela que los autores del campo infantil argentino tienen mucho para aportar sobre las formas de representar el mundo y dominan de manera original las formas estéticas desde las que buscan acercarlo al lector. Por otro lado, el sistema cultural tiene un compromiso pendiente con el campo infantil porque aún quedan por explorar en profundidad las posibilidades de acercar literatura e infancia desde las variables estéticas que rigen los textos del campo. En general, esta zona literaria es mirada con cierto prejuicio y el abordaje realizado hasta ahora parece responder a un interés particular o ser un gesto personal de ciertos autores, como es el caso de Walter Benjamin. Por último, resaltar los atributos de la colección como dispositivo de lectura deja afuera las oclusiones de este abordaje. Sin embargo, para mostrar las potencialidades del campo infantil inicialmente preferimos concentrarnos en este modo 
de leer que otorga autonomía al lector, implica realizar elecciones y tomar decisiones sabiendo que con ese gesto se postergan otras tantas lecturas y sentidos posibles.

\section{BibliografíA}

Benjamin, Walter (1989). Escritos. La literatura infantil, los niños y los jóvenes. Buenos Aires: Ediciones Nueva Visión.

Bornemann, Elsa (2004). ¡Socorro! (12 cuentos para caerse de miedo). Buenos Aires: Alfaguara.

Bornemann, Elsa (2008). Un elefante ocupa mucho espacio. Buenos Aires: Alfaguara.

Dalmaroni, Miguel (dir.) (2009). "1.Discusiones preliminares: el campo y el corpus", en La investigación literaria. Problemas iniciales de una práctica. Santa Fe: Ediciones UNL, 61-79.

Devetach, Laura (2008). Monigote en la arena. Buenos Aires: Ediciones Colihue.

Díaz Rönner, María A. (2000). "Literatura infantil de "menor" a "mayor", en Noe Jitrik (dir.), Historia Crítica de la literatura argentina, t. 11, Buenos Aires: Emecé, 511-531.

García, Laura R. (2010). "Memoria de elefantes para la violencia política", El bilo de la fábula. Revista anual del Centro de Estudios Comparados 8, Santa Fe: Universidad Nacional del Litoral, 75-84. (2012). "La poética de Gustavo Roldán: un colectivo de personajes" en Valeria Sardi y Cristina Blake (comp.), Un territorio en construcción: la literatura argentina para niños. La Plata: Universidad Nacional de La Plata, 133-143.

(2013). "Lo monstruoso en la literatura argentina para niños. Colección de lecturas para contar la violencia política”, Telar. Revista del Instituto Interdisciplinario de Estudios Latinoamericanos 13, Tucumán: Facultad de Filosofía y Letras-UNT. (En prensa)

Gerbaudo, Analía (2007). Derrida y la construcción de un nuevo canon crítico para las obras literarias. Córdoba: Jorge Sarmiento Editor-Universitaslibros/Ed. FFyH (UNC).

(2011). "El docente como autor del currículum: una reinstalación política y teórica necesaria”, en Analía Gerbaudo (dir.), La lengua y la literatura en la escuela secundaria. Santa Fe: Homo Sapiens Ediciones, 17-27.

Hassoun, Jacques (1996). Los contrabandistas de la memoria. Buenos Aires: Ediciones de la Flor.

Jackson, Rosmary (1986). Fantasy. Literatura y subversión. Buenos Aires: Catálogos.

Jelin, Elizabeth (2002). Los trabajos de la memoria, memorias de la represión. Buenos Aires: Siglo XXI Editores. 
Jelin, Elizabeth y Susana Kaufman (comp.) (2006), "Introducción”, en Subjetividad y figuras de la memoria. Buenos Aires: Siglo XXI Editores, 9-15.

Mariño, Ricardo (2008). "El genio del basural”, en El héroe y otros cuentos. Buenos Aires: Alfaguara Infantil.

Montes, Graciela (1991). Irulana y el ogronte (Un cuento de mucho miedo). Buenos Aires: Libros del Quirquincho.

- (2003). Tengo un monstruo en el bolsillo. Buenos Aires: Editorial Sudamericana.

— (2007). Otroso. Últimas noticias del mundo subterráneo. Buenos Aires: Alfaguara.

Nofal, Rossana (2006). "Literatura para chicos y memorias: colección de lecturas" en E. Jelin y S. Kaufman (comp.), Subjetividad y figuras de la memoria. Buenos Aires: Siglo XXI Editores, 111-129.

Ricoeur, Paul (1999). La lectura del tiempo pasado: memoria y olvido. Madrid: Arrecife.

Ritvo, Juan Bautista (1988). "Walter Benjamin y la retórica de la ciudad", Revista Paradoxa. Literatura/Filosofía 3 (3) (diciembre). Disponible en: http://bit.ly/15jcp2H

Roldán, Gustavo (1999). Probibido el elefante. Buenos Aires: Editorial Sudamericana.

— (2007a). Cada cual se divierte como puede. Buenos Aires: Ediciones Colihue.

— (2007b). Como si el ruido pudiera molestar. Buenos Aires: Editorial Norma.

- (2008a). El monte era una fiesta. Buenos Aires: Ediciones Colihue.

- (2008b). Sapo en Buenos Aires. Buenos Aires: Ediciones Colihue.

Schujer, Silvia (2006). Oliverio junta preguntas. Buenos Aires: Editorial Sudamericana.

Walsh, María Elena (2007). Dailan Kifki. Buenos Aires: Alfaguara.

Williams, Raymond (1980). Marxismo y literatura. Barcelona: Península.

Wolf, Ema (2011). Maruja. Buenos Aires: Editorial Sudamericana.

Para citar este artículo:

García, Laura Rafaela. 2016. "La colección como dispositivo de lectura de la violencia política en la literatura infantil argentina." Investigación Bibliotecológica: Archivonomía, Bibliotecología e Información 69: 263-284. http://dx.doi.org/10.1016/j.ibbai.2016.04.020 